\title{
SUPPLEMENTARY MATERIAL FOR: THE SCREENING AND RANKING ALGORITHM TO DETECT DNA COPY NUMBER VARIATIONS
}

\author{
By Yue S. Niu*, And Heping Zhang ${ }^{\dagger}$ \\ University of Arizona ${ }^{*}$ and Yale University ${ }^{\dagger}$
}

\section{APPENDIX A: GENERAL WEIGHT FUNCTIONS}

In this section, we consider a family of local diagnostic functions, which is the weighted average of $Y_{i}$ 's near the point of interest $x$

$$
D(x, h)=\sum_{i=1}^{n} w_{i}(x) Y_{i}
$$

where $w_{i}(x)=w_{i-x}$, and $\mathbf{w}=\left(\cdots, w_{-1}, w_{0}, w_{1}, \cdots\right)$, satisfying the following conditions:

1. local supportedness: $\exists$ an integer $h \ll n$, such that $w_{i}=0$ for $|i|>h$;

2. quasi-symmetry: $i \cdot w_{i} \leq 0$ and $\sum_{i \leq 0} w_{i}=-\sum_{i>0} w_{i}$ i.e. $\sum w_{i}=0$;

3. unity: $\sum_{i \leq 0}\left|w_{i}\right|=\sum_{i>0}\left|w_{i}\right|=1$, and hence $\|\mathbf{w}\|_{\ell_{1}}=\sum\left|w_{i}\right|=2$;

4. negligibility: $\|\mathbf{w}\|_{\ell_{2}}^{2} /\|\mathbf{w}\|_{\ell_{1}}^{2}=\sum w_{i}^{2} / 4=O\left(h^{-1}\right)$.

We denote by $\mathcal{W}$ the set of all weight vectors satisfying these four conditions. These four conditions are quite natural. The locally supported condition makes $D(x)$ depend on only those $Y_{i}$ 's within distance $h$. The quasisymmetric condition ensures that $D(x)$ measures the difference between the left-hand-side $Y_{i}^{\prime}$ 's and right-hand-side $Y_{i}$ 's. The unity condition is not essential, but helpful for easy presentation. The negligible condition, a little stronger than the traditional negligible condition, prevents the weights from concentrating on few points as the bandwidth $h$ tends to infinity. It is easy to see that all weights introduced in Section 2.2, up to a normalizing constant, are special cases of this family. Moreover, the SaRa with any local diagnostic function in this family satisfies the sure coverage property.

\section{APPENDIX B: PROOFS}

We shall prove Theorem 1 in three steps, represented by three lemmas. We introduce the notation and outline the proof first. 
A point $x$ is called $h$-flat if there is no change-point in the $h$-neighborhood of $x$, i.e. the interval $(x-h, x+h)$. We omit $h$ and say $x$ is a flat point if $h$ is obvious in the context. Let $\mathcal{F}_{h}$ be the set of all $h$-flat points of step function $\mu$. Consider the event $\mathcal{A}_{\tau}=\{|D(\tau, h)|>\lambda\}$ for change-point $\tau \in \mathcal{J}$ and the event $\mathcal{B}_{x}=\{|D(x, h)|<\lambda\}$ for flat point $x \in \mathcal{F}_{h}$. Define the event

$$
\mathcal{E}_{n}=\left(\bigcap_{\tau \in \mathcal{J}} \mathcal{A}_{\tau}\right) \bigcap\left(\bigcap_{x \in \mathcal{F}_{h}} \mathcal{B}_{x}\right) .
$$

In Lemma 1, we derive the distribution of $D(x, h)$ at a given point $x$ when there is no change-point other than possibly $x$ in the interval $(x-h, x+h)$. Then we calculate the probability $\mathbf{P}\left(\mathcal{E}_{n}\right)$ in Lemma 2 . In the final step, we show that $\mathcal{J} \subset: \hat{\mathcal{J}} \pm h$ holds under the event $\mathcal{E}_{n}$.

Lemma 1 If the noises are i.i.d. Gaussian, then for fixed $x$ and $h, D(x, h)$ is Gaussian. In particular, if $x$ is a flat point, $D(x, h) \sim \mathcal{N}\left(0, \Delta^{2}\right)$. If $\tau$ is a change-point with jump size $\delta, D(\tau, h) \sim \mathcal{N}\left(\delta, \Delta^{2}\right)$. Here,

$$
\Delta^{2}=\sum_{i} w_{i}^{2} \sigma^{2}=O\left(h^{-1}\right) \sigma^{2}
$$

Proof of Lemma 1. $D(x, h)$ is a linear combination of Gaussian variables, so it is Gaussian as well. It follows from the quasi-symmetric and unity conditions that the mean of $D(x, h)$ is zero for a flat point and $\delta$ for a change-point with jump size $\delta$. The variance is $\sum_{i} w_{i}^{2} \sigma^{2}$, which is of order $O\left(h^{-1}\right) \sigma^{2}$ by the condition 4 on the family $\mathcal{W}$. In particular, for the equally weighted case (2.1), $\Delta^{2}=\frac{2}{h} \sigma^{2}$.

Lemma 2 Under Assumption (3.1), there exist $h$ and $\lambda$ such that

$$
P\left(\mathcal{E}_{n}\right) \rightarrow 1 \text { as } n \rightarrow \infty .
$$

Proof of Lemma 2. It suffices to show that there exist $\lambda$ and $h$ such that

$$
\mathbf{P}\left(\mathcal{E}_{n}^{c}\right) \leq \mathbf{P}\left\{\bigcup_{\tau \in \mathcal{J}} \mathcal{A}_{\tau}^{c}\right\}+\mathbf{P}\left\{\bigcup_{x \in \mathcal{F}_{h}} \mathcal{B}_{x}^{c}\right\} \rightarrow 0 .
$$

Take $\lambda=\frac{1}{2} \delta$ and $h=\frac{1}{2} L$ where $\delta=\min \left|\delta_{j}\right|, L=\min _{1 \leq j \leq J+1}\left(\tau_{j}-\tau_{j-1}\right)$. By Lemma 1 , it is obvious that for each $\tau \in \mathcal{J}$ and $x \in \mathcal{F}_{h}, \mathbf{P}\left(\mathcal{A}_{\tau}^{c}\right)<1-\Phi\left(\frac{\delta}{2 \Delta}\right)$ and $\mathbf{P}\left(\mathcal{B}_{x}^{c}\right)=2\left(1-\Phi\left(\frac{\delta}{2 \Delta}\right)\right)$, where $\Phi$ is the cumulative distribution function of 
standard normal distribution. Note the following inequality for the Gaussian tail probability (Gordon, 1941)

$$
1-\Phi(t)<t^{-1} e^{-\frac{1}{2} t^{2}}
$$

By Bonferroni inequality and $\Delta=\sqrt{2 / h} \sigma=2 \sigma / \sqrt{L}$, we have

$$
\mathbf{P}\left(\mathcal{E}_{n}^{c}\right)<2 n \frac{2 \Delta}{\delta} e^{-\frac{\delta^{2}}{8 \Delta^{2}}}=\frac{8 n \sigma}{\delta \sqrt{L}} e^{-\frac{L \delta^{2}}{32 \sigma^{2}}}=\frac{8 n}{S} e^{-\frac{S^{2}}{32}} .
$$

It is guaranteed by Assumption (3.1) that the right hand side of (B.2) goes to zero as $n \rightarrow \infty$.

Lemma $3 \mathcal{J} \subset: \hat{\mathcal{J}} \pm h$ holds under event $\mathcal{E}_{n}$.

Proof of Lemma 3. We want to show that there is a one-to-one correspondence between $\mathcal{J}$ and $\hat{\mathcal{J}}$. Under event $\mathcal{E}_{n}$, no flat points can be selected into $\hat{\mathcal{J}}$ at the screening step. In other words, for any point $\hat{\tau} \in \hat{\mathcal{J}}$, there is at least one change-point in its $h$-neighborhood $(\hat{\tau}-h, \hat{\tau}+h)$. In fact, there is at most one such change-point by our assumption that $L=2 h$. Therefore, there is exactly one change-point within $(\hat{\tau}-h, \hat{\tau}+h)$ for each $\hat{\tau} \in \hat{\mathcal{J}}$. On the other hand, under event $\mathcal{E}_{n}$, for every change-point $\tau \in \mathcal{J}$, we have $|D(\tau, h)|>\lambda$. Moreover, $\tau-h$ and $\tau+h$ must be flat points since $L=2 h$. It follows that $\max \{|D(\tau-h, h)|,|D(\tau+h, h)|\}<\lambda$ and there is a local maximum, say $\hat{\tau}$, which is in $(\tau-h, \tau+h)$ and $|D(\hat{\tau}, h)| \geq|D(\tau, h)|>\lambda$.

\section{REFERENCES}

Gordon, R. D. (1941). Values of mills' ratio of area to bounding ordinate and of the normal probability integral for large values of the argument. The Annals of Mathematical Statistics 12, pp. 364-366.

Dr. Yue S. NiU

Department of Mathematics

The University of ARIZOnA

TuCSON AZ, 85721

E-MAIL: yueniu@math.arizona.edu
Professor Heping Zhang

Department of Epidemiology and

Public Health, Yale University

School of Medicine, New Haven CT, 06520

E-MAIL: heping.zhang@yale.edu 